\title{
Updated Assessment of the Association of the XRCC1 Arg399GIn Polymorphism with Lung Cancer Risk in the Chinese Population
}

\author{
Hai-Yan Yang ${ }^{1}$, Si-Yu Yang ${ }^{1}$, Fu-Ye Shao' ${ }^{1}$ Hai-Yu Wang ${ }^{2}$, Ya-Dong Wang2*
}

\begin{abstract}
Background: Published studies have reported relationships between $X$-ray repair cross-complementing group 1 (XRCC1) Arg399GIn polymorphism and lung cancer risk in Chinese population. However, the epidemiological results remained controversial. The objective of this study was to clarify the association of XRCC1 Arg399GIn polymorphism with lung cancer risk in the Chinese population. Materials and Methods: Systematic searches were performed through the database of Medline/Pubmed, Web of Science, Embase, CNKI and WanFang Medical Online. Odds ratios (ORs) with $95 \%$ confidence interval $(95 \% \mathrm{CI})$ were calculated to estimate the strength of the association. Results: Overall, we observed an increased lung cancer risk among subjects carrying XRCC1 codon $399 \mathrm{GIn} / \mathrm{GIn}$ genotype (OR=1.36, 95\% CI: 1.09-1.71) in the Chinese population on the basis of 19 studies with 5,416 cases and 5,782 controls. We did not observe any association between XRCC1 codon $399 \mathrm{Arg} / \mathrm{Gln}$ and $\mathrm{Arg} / \mathrm{Gln}+\mathrm{Gln} / \mathrm{Gln}$ polymorphisms and lung cancer risk $(\mathrm{OR}=1.00,95 \% \mathrm{CI}$ : 0.92-1.08 and $\mathrm{OR}=1.05,95 \% \mathrm{CI}$ : 0.97 1.13, respectively). Limiting the analysis to studies with controls in agreement with Hardy-Weinberg equilibrium (HWE), we observed an increased lung cancer risk among subjects carrying XRCC1 codon 399 GIn/Gln genotype $(\mathrm{OR}=1.18,95 \% \mathrm{CI}$ : 1.01-1.38). When stratified by source of control, we observed an increased lung cancer risk among subjects carrying XRCC1 codon $399 \mathrm{Arg} / \mathrm{Gln}+\mathrm{GIn} / \mathrm{Gln}$ genotype on the basis of hospitalized patient-based controls (OR=1.21, 95\% CI: 1.04-1.42) and among subjects carrying XRCC1 codon 399 GIn/Gln genotype on the basis of healthy subject-based controls $(\mathrm{OR}=1.22,95 \% \mathrm{CI}$ : 1.04-1.43). Conclusions: Our findings indicated that certain XRCC1 Arg399GIn variants might affect the susceptibility of lung cancer in Chinese population. Larger sample size studies are required to confirm our findings.
\end{abstract}

Keywords: Lung cancer - risk - XRCC1 Arg399Gln - polymorphism - meta-analysis - Chinese

Asian Pac J Cancer Prev, 16 (2), 495-500

\section{Introduction}

Lung cancer was the most commonly diagnosed cancer as well as the leading cause of cancer death in males globally, and among females, it was the fourth most commonly diagnosed cancer and the second leading cause of cancer death (Jemal et al., 2011). The global incidence of lung cancer is $1,608,800$ per year, with an annual mortality rate of $1,378,400$. For the year 2000, an estimated $85 \%$ of lung cancer in men and $47 \%$ of lung cancer in women is the consequence of tobacco smoking (Parkin et al., 2005). But the rest of lung cancers occur in non-smokers, which suggests that genetic factors might also play important roles in the development of lung cancer. Recently, common genetic polymorphisms in genes involved in DNA repair have been reported to be of importance in determining an individual's susceptibility (Yu et al., 2011; Kazma et al., 2012; Zhong et al., 2012; Wang et al., 2014).

$\mathrm{X}$-ray repair cross-complementing group 1 (XRCC1) is a major DNA repair protein involved in base excision repair (BER) and single-strand breaks (SSBs) repair and plays an important role in the maintenance of genomic integrity. A polymorphism of Arg399Gln (rs25487) has been identified in the XRCC1 gene, which leads to amino acid substitutions (exon 10, G-A) (Shen et al., 1998). This mutation may alter XRCC1 function, diminish repair kinetics and influence susceptibility to cancers. To date, a series of studies have reported the association of XRCC1 Arg399Gln polymorphism with lung cancer risk in Chinese population (Ratnasinghe et al., 2001; Chen et al., 2002; Song et al., 2004; Chan et al., 2005; Hu et al., 2005; Shen et al., 2005; Zhang et al., 2005a; Zhang et al., 2005b; Yu et al., 2006; Yin et al., 2007; Li et al., 2008; Su et al., 2008; Li et al., 2011; Qian et al., 2011; Du et al., 2012; Wang et al., 2012a; Guo et al., 2013; Ouyang et al., 2013; Du et al., 2014). However, the results from epidemiological studies have been conflictive rather than conclusive. Moreover, two meta-analyses have reported the association between XRCC1 Arg399GIn polymorphism and lung cancer risk 
in Chinese population (Zheng et al., 2009; Li et al., 2013). But, there are some key limitations in the previous metaanalyses. For example, several overlapping data (Li et al., 2005a; Li et al., 2005b; Li et al., 2005c; Hao et al., 2006; Yin et al., 2009) were not excluded from Li et al's study (Li et al., 2013) and one eligible paper published before 2013 (Ratnasinghe et al., 2001) was missing. For Zheng et al's paper (Zheng et al., 2009), four eligible papers published before 2008 (Ratnasinghe et al., 2001; Chan et al., 2005; Hu et al., 2005; Zhang et al., 2005b) were not included. Therefore, the conclusions from these two studies were not entirely reliable. In order to obtain a more precise estimation of this relationship, a meta-analysis including a total of 19 selected studies was performed, which may provide the more comprehensive evidence for the association of XRCC1 Arg399Gln polymorphism with lung cancer risk in Chinese population.

\section{Materials and Methods}

\section{Literature and methods}

Systematic searches were carried out through the database of Medline/Pubmed, Web of Science, Embase, WanFang Medical Online and Chinese National Knowledge Infrastructure (CNKI), covering all papers publishing before December 5, 2014 with the following search terms: "lung cancer" or "lung carcinoma" and "XRCC1 Arg399Gln" or "rs25487" and "Polymorphism" and "China" or "Chinese". Additional studies were identified by the references lists of the selected papers.

Data inclusion criteria: (a) Papers investigating lung cancer risk and XRCC1 Arg399Gln polymorphism; (b) Papers focusing on Chinese population; (c) Casecontrol studies and cohort studies; (d) Papers offering the sample size, odds ratio (OR) and their $95 \%$ confidence interval $(95 \% \mathrm{CI})$ or the information that can help infer the results. Accordingly, articles that could not offer essential information were excluded; reviews and repeated or overlapping literatures were excluded, too. For overlapping papers, the most recent population or publication including more information was included.

In total, twenty-seven published papers were identified with the association between XRCC1 Arg399Gln polymorphism and lung cancer risk in Chinese population. We reviewed all papers in the light of the criteria defined above and excluded two reviews (Zheng et al., 2009; Li et al., 2013) and six overlapping articles (Li et al., 2005a; Li et al., 2005b; Li et al., 2005c; Hao et al., 2006; Yin et al., 2009; Wang et al., 2012b). Therefore, 19 studies were determined to enter our study.

\section{Data extraction}

Two authors (Siyu Yang and Fuye Shao) tabulated the data first, and then inputted them into an electronic database independently. The following information was subtracted from each paper: authors' name, publishing year, area, source of controls, total number of cases and controls, genotype frequency of case and control, and stratified factors. Characteristics of individual study were summarized in Table 1.

\section{Quantitative data synthesis}

To estimate the association of XRCC1 Arg399Gln polymorphism with lung cancer risk in Chinese population, we conducted a meta-analysis of selected studies. Data were combined to calculate the summary OR and 95\%CI using a fixed-effects model and a random-effects model (DerSimonian et al., 1986). The Cochrane Q statistics test was used to assess the heterogeneity among studies. If the effects are assumed to be homogenous, the fixed-effects model is used. Otherwise, a random-effects model is used if the effects are heterogeneous. The funnel plot was drawn to assess publication bias visually. Egger's test and Begg's test were also used to assess the publication bias (Begg et al., 1994; Egger et al., 1997). We tested whether genotype frequencies of controls were in Hardy-Weinberg equilibrium (HWE) using the $\chi^{2}$ test.

All of the statistical analyses were performed by using Review Manager (Version 5.0.24, the Cochrane Collaboration) and STATA10.0 software package (Stata Corporation, College Station, Texas). All the tests were two-sided, a $P$ value $<0.05$ for any test or model was considered to be statistically significant.

\section{Results}

\section{Meta-analysis databases}

A database was established according to the extracted information from each article. All essential information was listed in Table 1, which showed first author, publishing year, area, source of control, number of case and control, stratified factors and $\mathrm{P}$ value of HWE. There were a total of 19 studies with 5, 416 cases and 5, 782 controls concerning the relationship between XRCC1 Arg399Gln polymorphism and lung cancer risk in Chinese population.

\section{Test of heterogeneity}

The heterogeneity of XRCC1 codon 399 Arg/Gln vs Arg/Arg, Gln/Gln vs Arg/Arg and Arg/Gln+Gln/Gln vs Arg/Arg was analyzed for 19 case-control studies. Our results showed that XRCC1 codon 399 Gln/Gln vs Arg/Arg for total population and hospitalized patientsbased control, and Arg/Gln+Gln/Gln vs Arg/Arg for adenocarcinomas had the heterogeneity with a $P$ value less than 0.05 (Table 2). Therefore, a random-effects model was used to analyze the summary odds ratios for them. A fixed-effects model was used to analyze the summary odds ratios for the rest.

\section{Quantitative data synthesis}

Overall, we observed an increased lung cancer risk among subjects carrying XRCC1 codon 399 Gln/Gln genotype $(\mathrm{OR}=1.36,95 \% \mathrm{CI}: 1.09-1.71)$ in Chinese population on the basis of 19 studies with 5,416 cases and 5, 782 controls (Figure 1A). We did not observe any association between XRCC1 codon 399 Arg/Gln and $\mathrm{Arg} / \mathrm{Gln}+\mathrm{Gln} / \mathrm{Gln}$ polymorphisms and lung cancer risk in Chinese population $(\mathrm{OR}=1.00,95 \% \mathrm{CI}: 0.92-1.08$ and $\mathrm{OR}=1.05$, 95\%CI: 0.97-1.13, respectively) (Figure 1B and $1 \mathrm{C}$ ). Limiting the analysis to the studies with controls in agreement with HWE, we observed an increased lung 
Table 1. General Information of Selected Articles

\begin{tabular}{llllrrrl}
\hline First author & Year & Area & Source of control & $\begin{array}{r}\text { Number } \\
\text { of case }\end{array}$ & $\begin{array}{c}\text { Number } \\
\text { of control }\end{array}$ & HWE & Stratified factors \\
\hline (Chan et al., 2005) & 2005 & Hong Kong & Healthy subjects & 75 & 162 & 0.879127 & \\
(Chen et al., 2002) & 2002 & Jiangsu & Healthy subjects & 103 & 99 & 0.853812 & \\
(Du et al., 2012) & 2012 & Shanghai & Healthy subjects & 100 & 100 & 0.000006 & Smoke and histological type \\
(Du et al., 2014) & 2014 & Shanghai & Healthy subjects & 120 & 120 & 0.000000 & Smoke and histological type \\
(Guo et al., 2013) & 2013 & Heilongjiang & Hospitalized patients & 684 & 602 & 0.005453 & \\
(Hu et al., 2005) & 2005 & Jiangsu & Healthy subjects & 710 & 710 & 0.679058 & \\
(Li et al., 2008) & 2008 & Liaoning & Hospitalized patients & 350 & 350 & 0.239615 & Histological type \\
(Li et al., 2011) & 2011 & Chongqing & Healthy subjects & 455 & 443 & 0.052370 & Smoke and histological type \\
(Ouyang et al., 2013) & 2013 & Hunan & Healthy subjects & 82 & 201 & 0.148702 & \\
(Qian et al., 2011) & 2011 & Tianjin & Healthy subjects & 581 & 603 & 0.411222 & \\
(Ratnasinghe et al., 2001) & 2001 & Yunnan & Healthy subjects & 107 & 208 & 0.572907 & \\
(Shen et al., 2005) & 2005 & Yunnan & Healthy subjects & 116 & 109 & 0.053219 & \\
(Song et al., 2004) & 2004 & Jiangsu & Hospitalized patients & 104 & 104 & 0.466350 & Smoke and histological type \\
(Su et al., 2008) & 2008 & Shanxi & Healthy subjects & 162 & 244 & 0.848338 & Histological type \\
(Wang et al., 2012a) & 2012 & Henan & Healthy subjects & 209 & 256 & 0.302191 & \\
(Yin et al., 2007) & 2007 & Liaoning & Hospitalized patients & 205 & 193 & 0.198358 & \\
(Yu et al., 2006) & 2006 & Hubei & Healthy subjects & 104 & 121 & 0.288300 & \\
(Zhang et al., 2005a) & 2005 & Henan & Healthy subjects & 149 & 157 & 0.853973 & Smoke \\
(Zhang et al., 2005b) & 2005 & Beijing & Healthy subjects & 1000 & 1000 & 0.079392 & Histological type \\
\hline
\end{tabular}

Table 2. Summary Odds Ratios of the Relation of XRCC1 Codon 339 Polymorphism to Lung Cancer Risk in Chinese Population

\begin{tabular}{|c|c|c|c|c|c|c|c|c|c|c|c|}
\hline \multirow[t]{2}{*}{ Genotype } & \multirow[t]{2}{*}{ Case/Control } & \multicolumn{3}{|c|}{ Heterogeneity test } & \multicolumn{2}{|c|}{ Hypothesis test } & \multirow[t]{2}{*}{ df } & \multicolumn{2}{|c|}{ Begg's test } & \multicolumn{2}{|c|}{ Egger's test } \\
\hline & & $Q$ & $P$ & OR $(95 \% \mathrm{CI})$ & $Z$ & $P$ & & $Z$ & $P$ & $t$ & $P$ \\
\hline \multicolumn{12}{|l|}{ Total } \\
\hline Arg/Gln vs Arg/Arg & $4943 / 5383$ & 21.13 & 0.27 & $1.00(0.92-1.08)$ & 0.03 & 0.98 & 18 & 0.49 & 0.624 & 0.45 & 0.657 \\
\hline Gln/Gln vs Arg/Arg & $3395 / 3576$ & 34.41 & 0.01 & $1.36(1.09-1.71)$ & 2.69 & 0.007 & 18 & 0.56 & 0.576 & 0.39 & 0.700 \\
\hline $\begin{array}{l}\text { Arg/Gln+Gln/Gln vs Arg/Arg } \\
\text { Stratification by HWE }\end{array}$ & $5416 / 5782$ & 26.36 & 0.09 & $1.05(0.97-1.13)$ & 1.23 & 0.22 & 18 & 1.12 & 0.263 & 1.07 & 0.298 \\
\hline \multicolumn{12}{|l|}{ Yes } \\
\hline Arg/Gln vs Arg/Arg & $4133 / 4600$ & 20.42 & 0.16 & $1.00(0.92-1.09)$ & 0.10 & 0.92 & 15 & 0.41 & 0.685 & 0.37 & 0.719 \\
\hline Gln/Gln vs Arg/Arg & $2271 / 3024$ & 23.69 & 0.07 & $1.18(1.01-1.38)$ & 2.13 & 0.03 & 15 & 0.59 & 0.558 & 0.15 & 0.881 \\
\hline $\mathrm{Arg} / \mathrm{Gln}+\mathrm{G} \ln / \mathrm{Gln}$ vs $\mathrm{Arg} / \mathrm{Arg}$ & $4512 / 4960$ & 22.40 & 0.1 & $1.03(0.95-1.12)$ & 0.74 & 0.46 & 15 & 0.59 & 0.558 & 0.59 & 0.564 \\
\hline \multicolumn{12}{|l|}{ Stratification by source of control } \\
\hline \multicolumn{12}{|l|}{ Healthy subjects-based control } \\
\hline $\mathrm{Arg} / \mathrm{Gln}$ vs Arg/Arg & $3710 / 4199$ & 11.33 & 0.66 & $0.96(0.87-1.05)$ & 0.92 & 0.36 & 14 & 0.30 & 0.767 & 0.28 & 0.784 \\
\hline Gln/Gln vs Arg/Arg & $2562 / 2781$ & 20.51 & 0.11 & $1.22(1.04-1.43)$ & 2.39 & 0.02 & 14 & 0.49 & 0.621 & 1.42 & 0.179 \\
\hline Arg/Gln+Gln/Gln vs Arg/Arg & $4073 / 4533$ & 16.91 & 0.26 & $1.00(0.92-1.09)$ & 0.07 & 0.94 & 14 & 1.09 & 0.276 & 0.83 & 0.420 \\
\hline \multicolumn{12}{|l|}{ Hospitalized patients-based control } \\
\hline Arg/Gln vs Arg/Arg & $1233 / 1184$ & 6.50 & 0.09 & $1.14(0.97-1.34)$ & 1.59 & 0.11 & 3 & 1.02 & 0.308 & 2.40 & 0.139 \\
\hline Gln/Gln vs Arg/Arg & $833 / 795$ & 8.97 & 0.03 & $1.46(0.75-2.86)$ & 1.12 & 0.26 & 3 & 1.70 & 0.089 & 3.64 & 0.068 \\
\hline Arg/Gln+Gln/Gln vs Arg/Arg & $1343 / 1249$ & 5.13 & 0.16 & $1.21(1.04-1.42)$ & 2.42 & 0.02 & 3 & 1.02 & 0.308 & 0.98 & 0.431 \\
\hline \multicolumn{12}{|l|}{ Stratification by smoking status } \\
\hline \multicolumn{12}{|l|}{ Smokers } \\
\hline $\mathrm{Arg} / \mathrm{G} \ln +\mathrm{G} \ln / \mathrm{G} \ln$ vs $\mathrm{Arg} / \mathrm{Arg}$ & $386 / 335$ & 1.19 & 0.55 & $0.97(0.72-1.30)$ & 0.21 & 0.84 & 2 & 1.04 & 0.296 & 12.94 & 0.049 \\
\hline \multicolumn{12}{|l|}{ Nonsmokers } \\
\hline Arg/Gln+Gln/Gln vs Arg/Arg & $276 / 322$ & 4.29 & 0.12 & $1.12(0.81-1.55)$ & 0.71 & 0.48 & 2 & 0.00 & 1.000 & 0.62 & 0.648 \\
\hline \multicolumn{12}{|l|}{ Stratification by histological type } \\
\hline \multicolumn{12}{|l|}{ Squamous cell carcinoma } \\
\hline Arg/Gln+Gln/Gln vs Arg/Arg & $508 / 1348$ & 6.06 & 0.05 & $1.10(0.89-1.35)$ & 0.86 & 0.39 & 2 & 0.00 & 1.000 & 2.96 & 0.208 \\
\hline \multicolumn{12}{|l|}{ Adenocarcinoma } \\
\hline $\mathrm{Arg} / \mathrm{Gln}+\mathrm{Gln} / \mathrm{Gln}$ vs $\mathrm{Arg} / \mathrm{Arg}$ & $798 / 2342$ & 16.00 & 0.007 & $1.12(0.81-1.53)$ & 0.68 & 0.49 & 5 & 0.38 & 0.707 & 0.03 & 0.974 \\
\hline
\end{tabular}

*HWE: Hardy-Weinberg equilibrium

cancer risk among subjects carrying XRCC1 codon 399 Gln/Gln genotype (OR=1.18, 95\%CI: 1.01-1.38) (Table 2 ). When stratified by source of control, we observed an increased lung cancer risk among subjects carrying XRCC1 codon $399 \mathrm{Arg} / \mathrm{Gln}+\mathrm{Gln} / \mathrm{Gln}$ genotype on the basis of hospitalized patients-based control (OR=1.21, 95\%CI: 1.04-1.42) and among subjects carrying XRCC1 codon $399 \mathrm{Gln} / \mathrm{Gln}$ genotype on the basis of healthy subjects-based control (OR=1.22, 95\%CI: 1.04-1.43) (Table 2). We did not observe any association between XRCC1 Arg399Gln polymorphism and lung cancer risk in Chinese population in the additional subgroup analyses by smoking status and histological type (Table 2 ). 
A

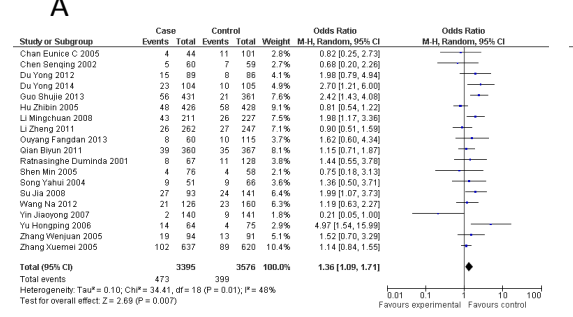

B

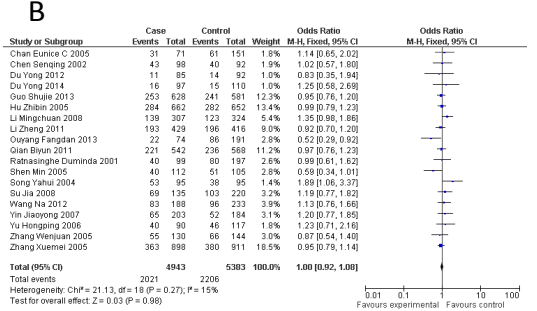

C

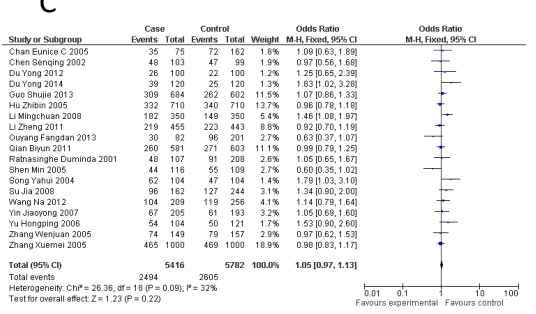

Figure 1. Forest Plots for the Association between XRCC1 Arg399GIn Polymorphism and Lung Cancer Risk in Chinese Population. A) Gln/Gln $v s \mathrm{Arg} / \mathrm{Arg}$; B) $\mathrm{Arg} / \mathrm{Gln} v s \mathrm{Arg} / \mathrm{Arg}$; C) $\mathrm{Arg} / \mathrm{Gln}+\mathrm{Gln} / \mathrm{Gln} v s \mathrm{Arg} / \mathrm{Arg}$ )
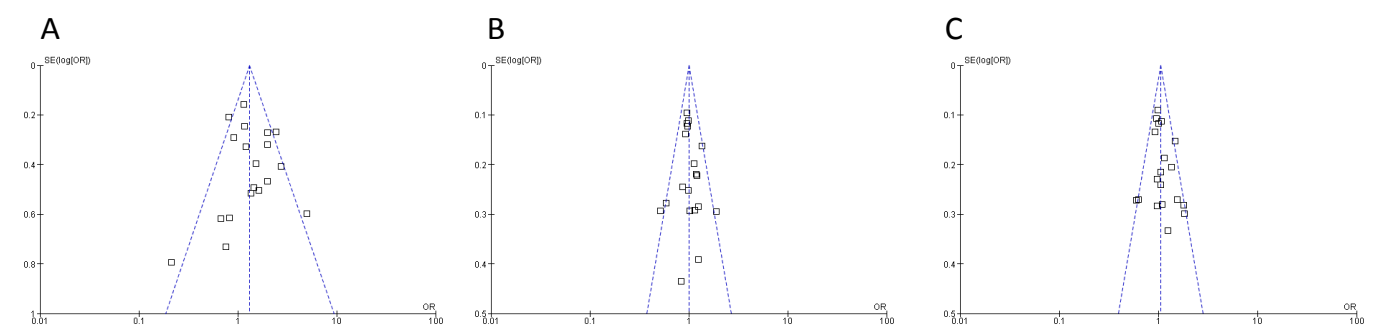

Figure 2. Funnel Plots for the Association between XRCC1 Arg399GIn Polymorphism and Lung Cancer Risk in Chinese Population in the Heterozygous Model. A) Gln/Gln $v s \mathrm{Arg} / \mathrm{Arg}$; B) $\mathrm{Arg} / \mathrm{Gln} v s \mathrm{Arg} / \mathrm{Arg}$; C) $\mathrm{Arg} / \mathrm{Gln}+\mathrm{Gln} /$ Gln $v s \mathrm{Arg} / \mathrm{Arg}$ )

\section{Bias diagnosis}

The shape of funnel plots seemed to be approximately symmetrical for Gln/Gln vs Arg/Arg, Arg/Gln vs Arg/ Arg and $\mathrm{Arg} / \mathrm{Gln}+\mathrm{Gln} / \mathrm{Gln}$ vs $\mathrm{Arg} / \mathrm{Arg}$ (Figure 2A, 2B and 2C). Egger's test and Begg's test suggested that publication biases may not have a significant influence on the results of the association between XRCC1 Arg399Gln polymorphism and lung cancer risk, except for XRCC1 codon $399 \mathrm{Arg} / \mathrm{Gln}+\mathrm{Gln} / \mathrm{Gln}$ vs $\mathrm{Arg} / \mathrm{Arg}$ in smokers since there was some uncertainty with the $\mathrm{P}$ value being equal to 0.049 in Egger's test (Table 2).

\section{Sensitivity analysis}

Sensitivity analyses were carried out to determine the effect of the individual study on the summary ORs by sequentially deleting each eligible study. The overall effects were not altered when the studies were homogenous for $\mathrm{Gln} / \mathrm{Gln} v s \mathrm{Arg} / \mathrm{Arg}$ among total population by deleting some studies (Data not shown).

\section{Discussion}

XRCC1 is an important component of the base excision repair system, which is a predominant DNA repair pathway for small base lesions resulting from oxidation and alkylation damage (Almeida et al., 2007). XRCC1 gene is mapped at human chromosome 19q13.213.3, which is 32.354 kilobases in length and consists of 16 introns and 17 exons. It encodes a 70-kDa scaffolding protein consisting of 633 amino acids, which coordinates a lot of protein-protein interactions, including DNA ligase III and DNA polymerase at the site of damage (Kubota et al., 1996; Vidal et al., 2001). It has been reported that more than 300 validated single nucleotide polymorphisms were identified in XRCC1 gene. Among them, XRCC1 Arg399Gln of exon 10 (rs25487) was the most extensively studied polymorphic site. Recently, meta-analysis studies have reported the association between XRCC1
Arg399Gln polymorphism and several kinds of cancer risk in Chinese population, such as colorectal cancer (Tian et al., 2013), esophageal cancer (Dai et al., 2009; Zhang et al., 2013) and hepatocellular carcinoma (Duan et al., 2012). In this study, we performed a systematic literature review to comprehensively evaluate the association between XRCC1 Arg399Gln polymorphism and lung cancer risk in Chinese population. We also estimated the possible effect modifications by source of control, smoking status, histological type and HWE in control. In summary, we observed an association of XRCC1 Arg399Gln polymorphism with lung cancer risk in Chinese population.

It is widely acknowledged that deviation from HWE may point to methodological weaknesses, such as biased selection of subjects or genotyping errors. The results of genetic association studies might be spurious if the distribution frequency of genotypes in the control group was not in agreement with HWE (Salanti et al., 2005). To address this issue, a stratified analysis was performed by HWE in control group in this study. When three studies (Du et al., 2012; Guo et al., 2013; Du et al., 2014) that significantly deviated from HWE were removed from this meta-analysis, no substantial modification of the results was observed, suggesting that this factor might not affect the combined effects in this current meta-analysis.

This meta-analysis should be expounded within the context of its limitations. Firstly, only published papers were included in this study, which may cause publication bias. To address this issue, Egger's test and Begg's test were performed. Our results signified that there was no significant publication bias in this study, except for subgroup analysis by smoking status. Secondly, each study had different eligibility criteria for subjects and different source of controls, which should be taken into consideration while interpreting the combined estimates. When subgroup analysis was performed by source of control, we observed an association between XRCC1 
Arg399Gln polymorphism and lung cancer risk among healthy subjects-based control. We also observed an association between XRCC1 Arg399Gln polymorphism and lung cancer risk among hospitalized patients-based control, but our results should be verified by larger sample size study, since only four studies were included in this meta-analysis. Thirdly, some factors such as sex, age, tobacco smoking, environment factors and histological type may influence the summary effects. We only performed subgroup analysis by smoking status and histological type in this study and did not observe any association between XRCC1 Arg399Gln polymorphism and lung cancer risk. Fourthly, but not the last one, the heterogeneity existed when comparing Gln/Gln genotype with Arg/Arg genotype in total population. To address this issue, sensitivity analysis was conducted. We still observed an increased lung cancer risk among subjects carrying XRCC1 codon $399 \mathrm{Gln} / \mathrm{Gln}$ genotype when the study was homogenous by removing some studies.

In conclusion, this systematic review demonstrates that XRCC1 Arg399Gln polymorphism appears to be a risk factor of lung cancer in Chinese population. Studies with large sample size are required to confirm the results from this current meta-analysis.

\section{References}

Almeida KH, Sobol RW (2007). A unified view of base excision repair: lesion-dependent protein complexes regulated by post-translational modification. DNA Repair (Amst), 6, 695-711.

Begg CB, Mazumdar M (1994). Operating characteristics of a rank correlation test for publication bias. Biometrics, $\mathbf{5 0}$, 1088-101.

Chan EC, Lam SY, Fu KH, et al (2005). Polymorphisms of the GSTM1, GSTP1, MPO, XRCC1, and NQO1 genes in Chinese patients with non-small cell lung cancers: relationship with aberrant promoter methylation of the CDKN2A and RARB genes. Cancer Genet Cytogenet, 162, 10-20.

Chen S, Tang D, Xue K, et al (2002). DNA repair gene XRCC1 and XPD polymorphisms and risk of lung cancer in a Chinese population. Carcinogenesis, 23, 1321-5.

Dai L, Wang K, Zhang J, et al (2009). XRCC1 gene polymorphisms and esophageal squamous cell carcinoma risk in Chinese population: A meta-analysis of case-control studies. Int J Cancer, 125, 1102-9.

DerSimonian R, Laird N (1986). Meta-analysis in clinical trials. Control Clin Trials, 7, 177-88.

Du Y, Chu DJ, Shi JM, et al (2012). The relationship between lung cancer and genetic polymorphism of XRCC1. J Clin Intern Med, 29, 346-8.

Du Y, He Y, Mei Z, et al (2014). Association between genetic polymorphisms in XPD and XRCC1 genes and risks of non-small cell lung cancer in East Chinese Han population. Clin Respir J.

Duan WH, Zhu ZY, Liu JG, et al (2012). XRCC1 Arg399Gln gene polymorphism and hepatocellular carcinoma risk in the Chinese Han population: a meta-analysis. Asian Pac J Cancer Prev, 13, 3601-4.

Egger M, Davey Smith G, Schneider M, et al (1997). Bias in meta-analysis detected by a simple, graphical test. $B m j$, 315, 629-34.

Guo S, Li X, Gao M, et al (2013). The relationship between
$\mathrm{XRCC} 1$ and XRCC3 gene polymorphisms and lung cancer risk in northeastern Chinese. PLoS One, 8, 56213.

Hao B, Miao X, Li Y, et al (2006). A novel T-77C polymorphism in DNA repair gene XRCC1 contributes to diminished promoter activity and increased risk of non-small cell lung cancer. Oncogene, 25, 3613-20.

$\mathrm{Hu} \mathrm{Z}$, Ma H, Lu D, et al (2005). A promoter polymorphism $(-77 \mathrm{~T}>\mathrm{C})$ of DNA repair gene XRCC1 is associated with risk of lung cancer in relation to tobacco smoking. Pharmacogenet Genomics, 15, 457-63.

Jemal A, Bray F, Center MM, et al (2011). Global cancer statistics. CA Cancer J Clin, 61, 69-90.

Kazma R, Babron MC, Gaborieau V, et al (2012). Lung cancer and DNA repair genes: multilevel association analysis from the International Lung Cancer Consortium. Carcinogenesis, 33, 1059-64.

Kubota Y, Nash RA, Klungland A, et al (1996). Reconstitution of DNA base excision-repair with purified human proteins: interaction between DNA polymerase beta and the XRCC1 protein. EMBO J, 15, 6662-70.

Li J, Mu L, Wei G, et al (2005a). Relationship between polymorphisms of DNA repair gene XRCC1 and susceptibility to lung cancer. China Oncology, 15, 335-8.

Li M, Yin Z, Cui Z, et al (2005b). Association of genetic polymorphism in DNA repair gene XRCC1 with risk of lung adenocarcinoma in nonsmoking women. Chinese $J$ Lung Cancer, 8, 431-4.

Li M, Yin Z, Guan P, et al (2008). XRCC1 polymorphisms, cooking oil fume and lung cancer in Chinese women nonsmokers. Lung Cancer, 62, 145-51.

Li MC, Cui ZS, He QC, et al (2005c). Association of genetic polymorphism in the DNA repair gene XRCC1 with susceptibility to lung cancer in non-smoking women. Chin J Oncol, 27, 713-6.

Li Y, Huang Y, Cao YS, et al (2013). Assessment of the association between XRCC1 Arg399Gln polymorphism and lung cancer in Chinese. Tumour Biol, 34, 3681-5.

Li Z, Guan W, Li MX, et al (2011). Genetic polymorphism of DNA base-excision repair genes (APE1, OGG1 and XRCC1) and their correlation with risk of lung cancer in a Chinese population. Arch Med Res, 42, 226-34.

Ouyang FD, Yang FL, Chen HC, et al (2013). Polymorphisms of DNA repair genes XPD, XRCC1, and OGG1, and lung adenocarcinoma susceptibility in Chinese population. Tumour Biol, 34, 2843-8.

Parkin DM, Bray F, Ferlay J, et al (2005). Global cancer statistics, 2002. CA Cancer J Clin, 55, 74-108.

Qian B, Zhang H, Zhang L, et al (2011). Association of genetic polymorphisms in DNA repair pathway genes with nonsmall cell lung cancer risk. Lung Cancer, 73, 138-46.

Ratnasinghe D, Yao SX, Tangrea JA, et al (2001). Polymorphisms of the DNA repair gene $\mathrm{XRCC} 1$ and lung cancer risk. Cancer Epidemiol Biomarkers Prev, 10, 119-23.

Salanti G, Amountza G, Ntzani EE, et al (2005). Hardy-Weinberg equilibrium in genetic association studies: an empirical evaluation of reporting, deviations, and power. Eur J Hum Genet, 13, 840-8.

Shen M, Berndt SI, Rothman N, et al (2005). Polymorphisms in the DNA base excision repair genes APEX1 and XRCC1 and lung cancer risk in Xuan Wei, China. Anticancer Res, 25, 537-42.

Shen MR, Jones IM, Mohrenweiser H (1998). Nonconservative amino acid substitution variants exist at polymorphic frequency in DNA repair genes in healthy humans. Cancer Res, 58, 604-8.

Song Y, Yin L, Pu Y, et al (2004). Relationship between Polymorphisms of DNA Repair Gene XRCC1 and 
Hai-Yan Yang et al

Susceptibility to Lung Cancer in Nanjing Population. Journal of Labour Medicine, 21, 18-21.

Su J, Niu R, Liu L, et al (2008). Regression analysis between polymorphisms of DNA repair gene XRCC1 and susceptibility to lung cancer of nonsmoking women. $J$ Zhengzhou University Medical Sciences, 43, 234-8.

Tian Z, Li YL, Liu JG (2013). XRCC1 Arg399Gln polymorphism contributes to increased risk of colorectal cancer in Chinese population. Mol Biol Rep, 40, 4147-51.

Vidal AE, Boiteux S, Hickson ID, et al (2001). XRCC1 coordinates the initial and late stages of DNA abasic site repair through protein-protein interactions. $E M B O J, \mathbf{2 0}$, 6530-9.

Wang L, Lin Y, Qi CC, et al (2014). Association of the XRCC1 c. $1178 \mathrm{G}>\mathrm{A}$ genetic polymorphism with lung cancer risk in Chinese. Asian Pac J Cancer Prev, 15, 4095-9.

Wang N, Wu Y, Zhou X (2012a). Association between genetic polymorphism of metabolizing enzymes and DNA repairing enzymes and the susceptibility of lung cancer in Henan population. Wei Sheng Yan Jiu, 41, 251-6.

Wang N, Wu YJ, Zhou XL, et al (2012b). The polymorphisms of XRCC1 gene and susceptibility to pulmonary cancer. Zhonghua Lao Dong Wei Sheng Zhi Ye Bing Za Zhi, 30,41-4.

Yin J, Vogel U, Ma Y, et al (2007). The DNA repair gene XRCC1 and genetic susceptibility of lung cancer in a northeastern Chinese population. Lung Cancer, 56, 153-60.

Yin J, Vogel U, Ma Y, et al (2009). Association of DNA repair gene XRCC1 and lung cancer susceptibility among nonsmoking Chinese women. Cancer Genet Cytogenet, 188, 26-31.

Yu H, Zeng X, Chou X, et al (2006). Polymorphism in the DNA repair gene XRCC1 and susceptibility to human lung cancer. J Guangxi Medical University, 23, 355-8.

Yu H, Zhao H, Wang LE, et al (2011). An analysis of single nucleotide polymorphisms of 125 DNA repair genes in the Texas genome-wide association study of lung cancer with a replication for the XRCC4 SNPs. DNA Repair, 10, 398-407.

Zhang W, Wu Y, Wu Y (2005a). Study on polymorphism of XRCC1 and susceptibility to lung cancer. China Public Health, 5, 561-3.

Zhang X, Miao X, Liang G, et al (2005b). Polymorphisms in DNA base excision repair genes ADPRT and XRCC1 and risk of lung cancer. Cancer Res, 65, 722-6.

Zhang ZY, Xuan Y, Jin XY, et al (2013). Meta-analysis demonstrates association of XRCC1 genetic polymorphism Arg399Gln with esophageal cancer risk in the Chinese population. Genet Mol Res, 12, 2567-77.

Zheng H, Wang Z, Shi X (2009). XRCC1 polymorphisms and lung cancer risk in Chinese populations: a meta-analysis. Lung Cancer, 65, 268-73.

Zhong D, Li G, Long J, et al (2012). The hOGG1Ser326Cys polymorphism and increased lung cancer susceptibility in Caucasians: an updated meta-analysis. Sci Rep, 2, 548. 